\title{
Use of the Faraday optical transformer for ultrafast magnetization reversal of nanomagnets
}

\author{
Volodymyr V. Kruglyak, ${ }^{a}$ Mikhail E. Portnoi, ${ }^{b}$ and Robert J. Hicken ${ }^{c}$ \\ ${ }^{a}$ School of Physics, University of Exeter, Stocker Road, Exeter EX4 4QL, United Kingdom \\ V.V.Kruglyak@exeter.ac.uk \\ ${ }^{\mathrm{b}}$ School of Physics, University of Exeter, Stocker Road, Exeter EX4 4QL, United Kingdom \\ M.E.Portnoi@exeter.ac.uk \\ ${ }^{c}$ School of Physics, University of Exeter, Stocker Road, Exeter EX4 4QL, United Kingdom \\ R.J.Hicken@exeter.ac.uk
}

\begin{abstract}
We propose a new strategy for ultrafast magnetization reversal of nanomagnets. Due to the Inverse Faraday Effect, circularly polarized optical pulses induce a pulsed magnetic flux in materials with large magneto-optical susceptibility. Alternatively, intense optical pulses can induce a pulsed magnetic flux by means of ultrafast demagnetization of a metallic thin film or multilayer with a perpendicular magnetic anisotropy. The time varying magnetic flux induces a transient electro-motive force and electric current in a conducting loop on the surface of the illuminated material, and hence a transient magnetic field. The magnetic field pulses due to the transient current appear to be too short for use in the magnetic field or spin-current induced precessional switching of magnetization. However, our calculations suggest that the magnetic field could lead to ultrafast switching of a nanomagnet overlaid on the surface of the conductor and demagnetized by the same optical pulse. In the case of magnetic pulses due to the Inverse Faraday Effect, the switching direction could be controlled by the helicity of the optical pulse.
\end{abstract}

Keywords: ultrafast phenomena, optoelectronics, magnetic properties.

\section{INTRODUCTION}

As magnetic data storage densities increase, there is a quest for faster methods of manipulating the magnetization of nanoscale elements within magnetic recording heads, patterned recording media, and magnetic random access memory (MRAM) devices. Several approaches to this task have been proposed. In precessional magnetization reversal, a pulsed magnetic field is applied perpendicularly to the static magnetization and terminated after a half period of the induced large amplitude magnetization precession, when the new equilibrium orientation is reached [1-3]. In the spin-torque reversal, electrical current is spin polarized in a thicker magnetic electrode and is used to exert a torque upon the magnetization of a thinner magnetic electrode [4-6]. The speed of these methods is limited by the frequency of the uniform ferromagnetic resonance and ability to generate sufficiently short magnetic field pulses. The nonuniformity of the magnetic response within the nanomagnetic elements may present an additional complication for the practical implementation of these schemes of magnetization reversal [7]. A faster method is based upon the phenomenon of ultrafast demagnetization [8], in which the sample magnetization collapses following absorption of an intense optical pulse and then recovers in the direction of the external magnetic field [9]. The speed of this mechanism is limited by the cooling time of the system and by the duration of the external magnetic field pulse.

The fact that circularly polarized light can induce a magnetization in a solid is referred to as the inverse Faraday effect and has been known for some time [10-12]. In optical pumpprobe experiments, it is known to be responsible for the third order non-linearity [13-15], and has been used to study the relaxation of the electron linear and angular momentum [16]. It 
was proposed that the transient magnetic field associated with circularly polarized laser pulses would be used in all-optical switching of magnetic elements $[17,18]$. The experimental evidence for the existence of such a field was presented in Refs. 19-21, where it was argued that femtosecond magnetic field pulses with magnitude of up to $5 \mathrm{~T}$ might be generated in this way. These fields were used to induce transient switching of the sample magnetization through an angle of $0.6^{\circ}$ [20], and to study spin wave dynamics at nearly $\mathrm{THz}$ frequencies in ferrimagnets and antiferromagnets $[22,23]$.

Little attention has been paid in the literature to the method by which optically generated magnetization was detected in Ref. 11, where an electrical current induced in a coil by the associated time dependent magnetic flux was measured. Based on the latter idea, we showed that subpicosecond pulses of magnetic field could be generated in this way [24]. Combining results from Refs. 9,19,24, here we propose a scheme for optically induced transient magnetic field assisted full magnetization reversal of nanoscale magnetic elements [25]. The scheme employs the transient magnetic field generated by the electrical current induced in a conducting ring underneath a magnetic element due to the optically induced pulsed magnetic flux through the area of the ring, and the ultrafast demagnetization of the magnetic element. The former mechanism of electrical current generation in a nanoscale conductor is based upon the inverse Faraday effect and Faraday's law of electromagnetic induction. Therefore we suggest that the proposed device be called a "Faraday optical transformer".

\section{THEORY}

In an ordinary electrical transformer, two coils are wound onto a ferromagnetic core. An AC electrical current in one of the coils then creates a time varying magnetic flux in the core. According to the Faraday's law of electro-magnetic induction, the time varying magnetic flux induces an electromotive force (e.m.f) and hence an electrical current in the other coil. On the other hand, Kimel et al demonstrated that due to the inverse Faraday effect circularly polarized optical pulses can induce very short (200 fs) pulses of large magnetic field in dysprosium orthoferrite $\left(\mathrm{DyFeO}_{3}\right)$ crystals [19], and hence magnetic flux pulses of the same duration and temporal shape. According to Faraday's law of electro-magnetic induction, the latter flux must induce an e.m.f. in a conducting loop deposited upon the crystal surface as shown in Fig. 1 (a).

Indeed, let us assume that the optical pulses, and hence the magnetic pulses optically induced in $\mathrm{DyFeO}_{3}$ crystal, have a Gaussian temporal form (Fig. 1 (c))

$$
B(t)=B_{0} e^{-\frac{t^{2}}{2 \sigma^{2}}},
$$

where $t$ is time and the full width at half maximum of the pulse is given by $2 \sigma \sqrt{\ln 2}$. Due to the continuity of the normal component of the magnetic induction at the crystal surface and tiny spontaneous magnetization $\left(M_{\mathrm{s}}=8 \mathrm{G}\right)$ of $\mathrm{DyFeO}_{3}$ [19], the field in the ring deposited upon the $\mathrm{DyFeO}_{3}$ crystal will be virtually the same as that at the surface of the crystal. The transient e.m.f. associated with the change of the magnetic flux through the area of the ring is then given by the Faraday's law of electromagnetic induction

$$
\varepsilon(t)=-\frac{\partial\left(B_{0} S\right)}{\partial t}=\frac{\varepsilon_{0} t}{\sigma} e^{-\frac{t^{2}}{2 \sigma^{2}}},
$$

where $S$ is the area of the ring, and $\varepsilon_{0}=B_{0} S / \sigma$. If the ring size is small compared to both the wavelength of light and the wavelength associated with the pulsed electromagnetic field in the ring [26], the ring can be considered as an $L R$ circuit (Fig. 1 (b)) with e.m.f. given by equation 

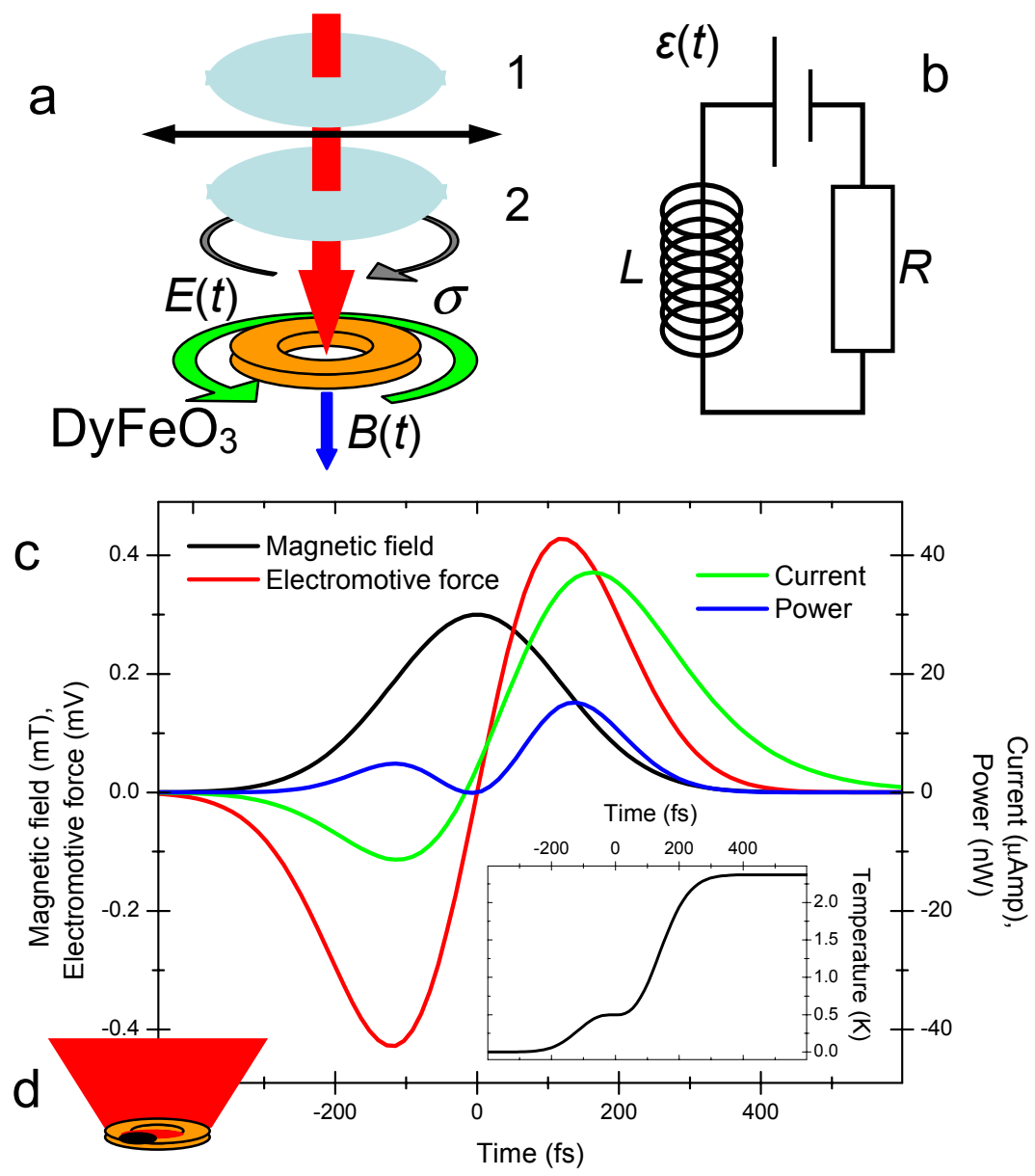

Fig. 1. (a) The scheme for transient magnetic field generation is shown. A laser pulse is circularly polarized after passing through the linear polarizer (1) and the quarterwave plate (2), and induces a time varying magnetic field $B(t)$ in the $\mathrm{DyFeO}_{3}$ crystal. The associated time varying magnetic flux through the area of the gold ring induces a time varying electric field $E(t)$, and hence e.m.f. (b) An equivalent $L R$ circuit is shown. (c) The time dependence of the transient magnetic field (left axis), e.m.f. (left axis), current (right axis) and electric power (right axis) is shown. In the calculation, we assumed that the ring had a radius of $300 \mathrm{~nm}$ and a circular crosssection with a radius of $30 \mathrm{~nm}$. The optical pulse was assumed to have a full width at half maximum of $200 \mathrm{fs}$ and an intensity that leads to a transient magnetic field with a peak value of $0.3 \mathrm{mT}$. The inset shows the transient temperature increase due to the dissipation of the electrical energy. (d) The geometry for the proposed magnetization reversal scheme, when the optical spot covers the ring with a nanomagnetic element on its surface, is shown schematically.

(2). Using the Kirchoff's law, the current in the circuit can be calculated analytically by direct integration, and is equal 


$$
I(t)=-\frac{B_{0} S}{L}\left(e^{-\frac{t^{2}}{2 \sigma^{2}}}-\frac{\sigma \sqrt{\pi}}{\tau}\left(1+\operatorname{erf}\left(\frac{t}{\sigma \sqrt{2}}-\frac{\sigma}{\tau \sqrt{2}}\right)\right) e^{\frac{\sigma^{2}}{2 \tau^{2}}-\frac{t}{\tau}}\right),
$$

where $\tau$ is the circuit time constant equal to $L / R$, and $L$ and $R$ are the self-inductance and resistance of the ring, respectively. Assuming that the ring has a radius $r_{1}$ and its cross-section is circular with radius $r_{2}$, one can obtain for its resistance and self-inductance [27]

$$
R=\frac{2 \rho r_{1}}{r_{2}^{2}}, L=\mu_{0} r_{1}\left(\ln \frac{8 r_{1}}{r_{2}}-\frac{7}{4}\right)
$$

where $\mu_{0}$ is the permeability of vacuum, and $\rho$ is the resistivity. The time dependence of the transient current in the ring (3)-(4) is shown in Fig. 1 (c). When scaled so as to have nanosecond duration, the current resembles qualitatively the signal measured in Ref. 11 in response to optical pulses of nanosecond rather than femtosecond duration.

\section{DISCUSSION}

At the surface of the ring, the current generates a magnetic field parallel to the surface and with magnitude given approximately by

$$
B_{\text {induced }}=\frac{\mu_{0} I(t)}{2 \pi r_{2}},
$$

The transient magnetic field $B_{\text {induced }}$ corresponding to the maximum current in Fig. 1 is about $0.25 \mathrm{mT}$. At the same time, a field of about $100 \mathrm{~T}$ is required for the precessional magnetization reversal of a magnetic element within $200 \mathrm{fs}$. In addition, the current pulse is bipolar, which does not favor this particular switching mechanism. Due to the very short duration, the current pulses are also unsuitable for spin torque magnetization reversal, although the generated current densities may be sufficiently large. Note that an increase of the pulse duration will result in a drastic reduction of the transient current magnitude, since the latter is proportional to the time derivative of the transient magnetic field. Nonetheless, the effect could be used within a magnetization reversal scheme that employs the ultrafast demagnetization effect.

Indeed, let us assume that the laser spot has the same size as the ring or is slightly larger. The laser pulse can then cause the magnetization of a magnetic element deposited on the surface of the ring to collapse within the duration of the optical pulse [28]. The magnetization of the element will then be restored in the direction of the magnetic field applied as the element cools. The right hand side of equation (3) contains two terms: a Gaussian (i. e. the temporal shape of the laser pulse) and a convolution of the Gaussian with the Heaviside step function and an exponential decay, with time constant equal to that of the $L R$ circuit. The magnetic field during the magnetization collapse, i. e. the Gaussian term in the right hand side of equation (3), is not important. However, the second term in the right hand side of equation (3) is delayed with respect to the Gaussian, and the delay increases with increasing the $L / R$ time constant. Therefore, the associated magnetic field can serve as the delayed nucleation field for the reset of the element magnetization.

The size of the device is determined by the minimum achievable optical spot size. The latter size scales approximately with the optical wavelength, which is several hundreds of nanometers in the visible range. An even smaller spot size could perhaps be achieved using near field optics, although the control of the optical polarization may be non-trivial in this regime. As shown in Figure 2, the peak current of the device appears to increase as the ring 


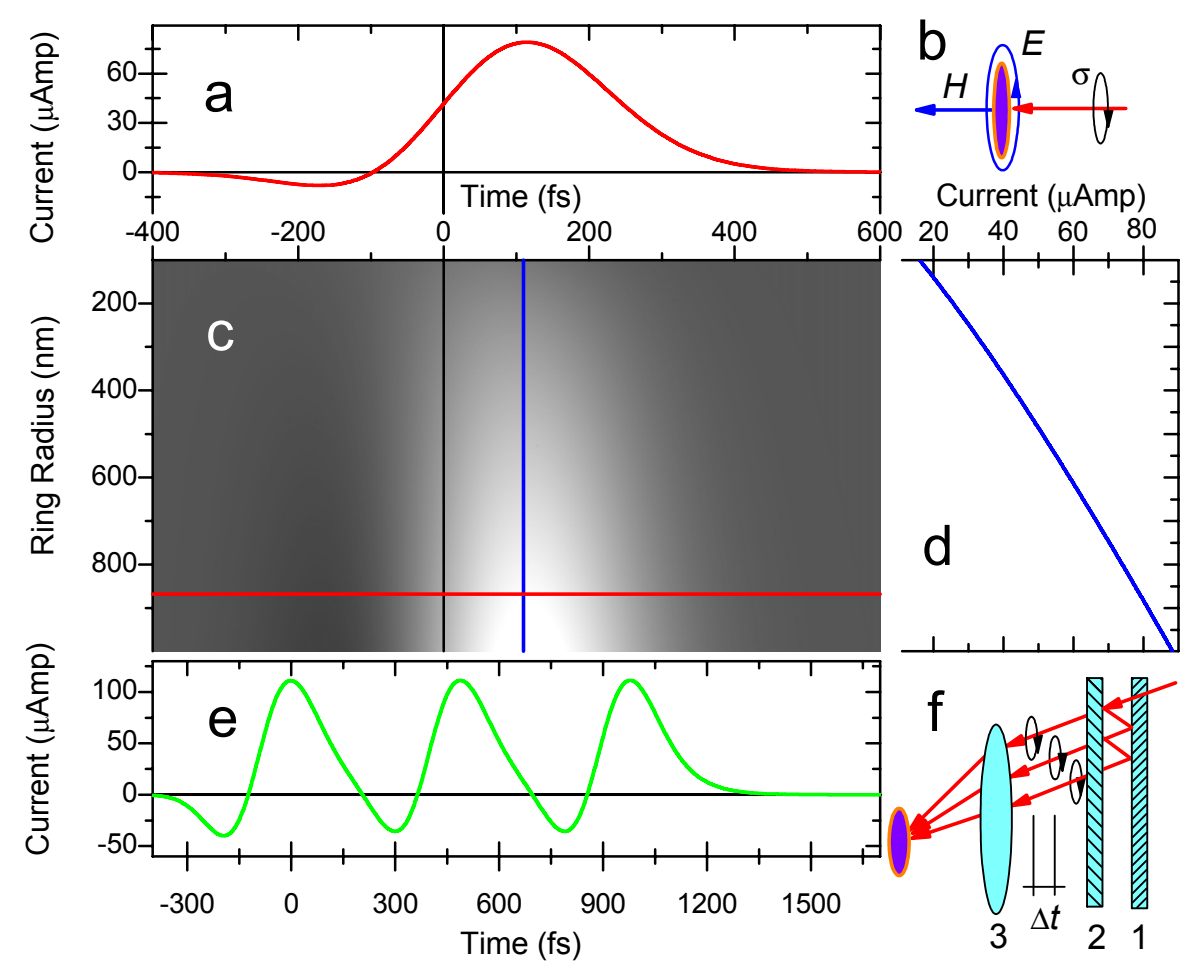

Fig. 2. (a), (c), and (d) The dependence of the current in the ring upon the ring radius and the time is presented for the experimental configuration schematically shown in (b). (e) The dependence of the current in the ring upon time is presented for the experimental configuration schematically shown in (f). The mirror 1 and the partially transmitting mirror 2 form a Fabry-Perot like assembly. 3 is a lens. The frequency of the ac current in (e) is $2 \mathrm{THz}$.

size increases. However, note that the coupling of the optically induced transient magnetic field in the core material may become weaker when the ring size becomes comparable to the associated electromagnetic wave wavelength.

The inset to Figure 1 (c) shows the calculated transient temperature increase due to the Joule heating in the ring due to a single laser pulse. The Joule heating was calculated by a numerical integration of the product of the transient e.m.f. and the electrical current shown in Figure $1(\mathrm{c})$. While the temperature increase appears to be quite moderate for the parameters used in the calculation, the performance of the proposed scheme is still limited by the need for an efficient heat sink for the optical energy required to induce the ultrafast demagnetization [9].

Estimations show that the thermal noise and the discrete electron current noise in such nano-scale devices are just an order of magnitude smaller than corresponding signal levels in the calculations presented here. At the same time, the excitation strength in our calculations was deliberately chosen to be quite modest, which is due to the shortage of experimental data in this immature field of investigations, while stronger excitation should be possible, leading to better signal-to-noise ratios. Further work both theoretical and experimental is required to improve the design and to finally implement the idea in a real device. 
Let us now discuss some implications of the experimental realization of the proposed Faraday optical transformer for other fields of physics and technology. Firstly, the Faraday optical transformer could be used in magnetic field pumped time resolved experiments as a photo-inductive rather than a photo-conductive switch [29]. Secondly, several optical pulses time delayed with respect to one another by a few hundreds of femtoseconds as shown in Figure 2 will lead to an electrical current modulated at a THz frequency. In this regime, the Faraday optical transformer will act as an emitter of pulsed $\mathrm{THz}$ radiation. Indeed, $\mathrm{THz}$ emission following an excitation of a magnetic metal film by an intense laser pulse was observed in Ref. 30. This implies that the core of the proposed devices could be made of a magnetic metal rather than dielectric, so that the transient flux change is achieved via the ultrafast demagnetization rather than the inverse Faraday effect. However, in this case, the direction of the generated magnetic field could not be varied by changing the helicity of the optical pulse. Thirdly, the local generation of the AC e.m.f. could be used to power nanoelectromechanical systems (NEMS). Finally, it would be interesting to know what happens if the conducting ring within the Faraday optical transformer is actually made of a superconductor material.

\section{SUMMARY}

In summary, we have proposed a scheme for optically induced and photo-magnetic field assisted magnetization reversal of nanomagnetic elements. The proposed device converts pulsed light into sub-picosecond electrical pulses and so can also be considered as an ultrafast optical transformer (Faraday optical transformer). Many potential applications can be foreseen in various fields of physics. These include but are not limited to NEMS, spintronics, terahertz radiation emission, and ultrafast optics.

In the first demonstration of the ultrafast precessional magnetization reversal, a $50 \mathrm{GeV}$ electron bunch from a particle accelerator was used to deliver a fast magnetic field pulse to a magnetic film [2]. Part of the film was inevitably destroyed in the experiment. The delivery of fast electrical and magnetic fields by means of the method proposed here suggests a far less violent means of controlling future nanoscale devices.

\section{Acknowledgments}

The authors gratefully acknowledge the financial support of the New Energy and Industrial Technology Development Organization (NEDO) and the UK Engineering and Physical Sciences Research Council (EPSRC).

\section{References}

[1] P. R. Gillette and K. Oshima, "Magnetization reversal by rotation," J. Appl. Phys. 29, 529-531 (1958) [doi:10.1063/1.1723211].

[2] H. C. Siegmann, E. L. Garwin, C. Y. Prescott, J. Heidmann, D. Mauri, D. Weller, R. Allenspach, and W. Weber, "Magnetism with picosecond field pulses," J. Magn. Magn. Mater. 151, L8-L12 (1995) [doi:10.1016/0304-8853(95)00602-8].

[3] T. Gerrits, H. A. M. van den Berg, J. Hohlfeld, L. Bär, and T. Rasing, "Ultrafast precessional magnetization reversal by picosecond magnetic field pulse shaping," Nature 418, 509-512 (2002) [doi:10.1038/nature00905].

[4] L. Berger, "Emission of spin waves by a magnetic multilayer traversed by a current," Phys. Rev. B 54, 9353-9358 (1996) [doi:10.1103/PhysRevB.54.9353].

[5] J. C. Slonczewski, "Current-driven excitation of magnetic multilayers," J. Magn. Magn. Mater. 159, L1-L7 (1996) [doi:10.1016/0304-8853(96)00062-5]. 
[6] J. A. Katine, F. J. Albert, R. A. Buhrman, E. B. Myers, and D. C. Ralph, "Currentdriven magnetization reversal and spin-wave excitations in $\mathrm{Co} / \mathrm{Cu} / \mathrm{Co}$ pillars," Phys. Rev. Lett. 84, 3149-3152 (2000) [doi:10.1103/PhysRevLett.84.3149].

[7] V. V. Kruglyak, A. Barman, R. J. Hicken, J. R. Childress and J. A. Katine, "Picosecond magnetization dynamics in nanomagnets: Crossover to nonuniform precession," Phys. Rev. B 71, 220409 (2005) [doi:10.1103/PhysRevB.71.220409].

[8] E. Beaurepaire, J.-C. Merle, A. Daunois, and J.-Y. Bigot, "Ultrafast spin dynamics in ferromagnetic Nickel," Phys. Rev. Lett. 76, 4250-4253 (1996) [doi:10.1103/PhysRevLett.76.4250].

[9] J. Hohlfeld, T. Gerrits, M. Bilderbeek, T. Rasing, H. Awano, and N. Ohta, "Fast magnetization reversal of GdFeCo induced by femtosecond laser pulses," Phys. Rev. $B$ 65, 012413 (2002) [doi:10.1103/PhysRevB.65.012413].

[10] L. P. Pitaevskii, "Electric forces in a transparent dispersive medium," Sov. Phys. JETP 12, 1008-1013 (1961).

[11] J. P. van der Ziel, P. S. Pershan, and L. D. Malmstrom, "Optically-induced magnetization resulting from the inverse Faraday effect," Phys. Rev. Lett. 15, 190193 (1961) [doi:10.1103/PhysRevLett.15.190].

[12] R. Hertel, "Theory of the inverse Faraday effect in metals," J. Magn. Magn. Mater. 303, L1-L4 (2006) [doi:10.1016/j.jmmm.2005.10.225].

[13] Y. P. Svirko and N. I. Zheludev, Polarization of Light in Nonlinear Optics, Wiley, New York (1998).

[14] N. I. Zheludev, P. J. Bennett, H. Loh, S. V. Popov, I. R. Shatwell, Y. P. Svirko, V. E. Gusev, V. F. Kamalov, and E. V. Slobodchikov, "Cubic optical nonlinearity of free electrons in bulk gold," Opt. Lett. 20, 1368-1370 (1995).

[15] V. V. Kruglyak, R. J. Hicken, M. Ali, B. J. Hickey, A. T. G. Pym, and B. K. Tanner, "Ultrafast third-order optical nonlinearity of noble and transition metal thin films," J. Opt. A: Pure Appl. Opt. 7, S235 (2005) [doi:10.1088/1464-4258/7/2/031].

[16] V. V. Kruglyak, R. J. Hicken, M. Ali, B. J. Hickey, A. T. G. Pym, and B. K. Tanner, "Measurement of hot electron momentum relaxation times in metals by femtosecond ellipsometry," Phys. Rev. B 71, 233104 (2005) [doi:10.1103/PhysRevB.71.233104].

[17] Y. I. Gorobets', Y. I. Dzhezherya, and A. F.Kravets, "Magnetization reversal of ferromagnetic granules by field of circularly-polarized electromagnetic waves," Metallofiz. Nov. Tekhn. 25, 27-36 (2003).

[18] R. Gomez-Abal, O. Ney, K. Satitkovitchai, and W. Hübner, "All-optical subpicosecond magnetic switching in $\mathrm{NiO}(001)$," Phys. Rev. Lett. 92, 227402 (2004) [doi:10.1103/PhysRevLett.92.227402].

[19] A. V. Kimel, A. Kirilyuk, P. A. Usachev, R. V. Pisarev, A. M. Balbashov, T. Rasing, "Ultrafast non-thermal control of magnetization by instantaneous photomagnetic pulses," Nature 435, 655-657 (2005) [doi:10.1038/nature03564].

[20] F. Hansteen, A. Kimel, A. Kirilyuk, and T. Rasing, "Femtosecond photomagnetic switching of spins in ferrimagnetic garnet films," Phys. Rev. Lett. 95, 047402 (2005) [doi:10.1103/PhysRevLett.95.047402].

[21] F. Hansteen, A. Kimel, A. Kirilyuk, and T. Rasing, "Nonthermal ultrafast optical control of the magnetization in garnet films," Phys. Rev. B 73, 014421 (2006) [doi:10.1103/PhysRevB.73.014421].

[22] C. D. Stanciu, A. V. Kimel, F. Hansteen, A. Tsukamoto, A. Itoh, A. Kirilyuk, and T. Rasing, "Ultrafast spin dynamics across compensation points in ferrimagnetic GdFeCo: The role of angular momentum compensation," Phys. Rev. B 73, 220402 (2006) [doi:10.1103/PhysRevB.73.220402].

[23] A. V. Kimel, C. D. Stanciu, P. A. Usachev, R. V. Pisarev, V. N. Gridnev, A. Kirilyuk, and T. Rasing, "Optical excitation of antiferromagnetic resonance in TmFeO 3 ," Phys. Rev. B 74, 060403 (2006) [doi:10.1103/PhysRevB.74.060403]. 
[24] V. V. Kruglyak and M. E. Portnoi, "Generation of femtosecond current pulses using the inverse magneto-optical Faraday effect," Techn. Phys. Lett. 31, 1047-1048 (2005) [doi:10.1134/1.2150894].

[25] V.V. Kruglyak, M.E. Portnoi, and R.J. Hicken, "Generation of femtosecond electromagnetic pulses at the nanoscale", Proc. SPIE 6328, 63280K (2006) [doi: $10.1117 / 12.677370]$.

[26] For the 200 fs pulses from Ref. 19, the associated electromagnetic wavelength is about $60 \mu \mathrm{m}$.

[27] L. D. Landau and E. M. Lifshitz, Electrodynamics of continuous media, Pergamon, Oxford (1984).

[28] L. Guidoni, E. Beaurepaire, and J.-Y. Bigot, "Magneto-optics in the ultrafast regime: Thermalization of spin populations in ferromagnetic films," Phys. Rev. Lett. 89, 017401 (2002) [doi:10.1103/PhysRevLett.89.017401].

[29] M. R. Freeman, R. R. Ruf, and R. J. Gambino, "Picosecond pulsed magnetic fields for studies of ultrafast magneticphenomena," IEEE Trans. Magn. 27, 4840-4842 (1991) [doi:10.1109/20.278964].

[30] E. Beaurepaire, G. M. Turner, S. M. Harrel, M. C. Beard, J.-Y. Bigot and C. A. Schmuttenmaer, Appl. Phys. Lett. 84, 3465-3467 (2004) [doi:10.1063/1.1737467].

Volodymyr V. Kruglyak is a research fellow at the University of Exeter. He received his undergraduate degrees in theoretical physics from Donetsk National University in 2001, and his $\mathrm{PhD}$ degree in experimental physics from the University of Exeter in 2004. He is the author of more than 30 journal papers. His current research interests include nanomagnetism and magnonics, ultrafast magneto-optics, spintronics, magnetoelectronic and optoelectronic systems.

Mikhail E. Portnoi is a Senior Lecturer in Theoretical Physics at the University of Exeter. $\mathrm{He}$ obtained his MSc degree in electrical engineering from the Leningrad Electrotechnical Institute in 1988, his Candidate of Physical-Mathematical Sciences degree from the A.F. Ioffe Physical-Technical Institute in 1994, and PhD in physics from the University of Utah in 1996. $\mathrm{He}$ is the author of more than 50 journal papers. His research interests include carbon nanotubes, excitons and electron-hole plasma in semiconductor quantum wells, $\mathrm{THz}$ optoelectronics, optical spin orientation in nanostructures and the quantum Hall effect.

Robert J. Hicken is a Professor of Physics at the University of Exeter. He received his BA degree in physics from the University of Oxford in 1986, and his MA and PhD degrees from Johns Hopkins University in 1988 and 1991, respectively. He is the author of more than 60 journal papers. His current research interests include magnetism, spintronics and non-linear optics. 